\title{
Aproximación al Origen de la Noción de Objeto de Aprendizaje: Revisión Histórico - Bibliográfica
}

\section{Approach to the Origin of the Notion of Learning Object: Historical and Bibliographic Review}

\author{
DOI: http://dx.doi.org/10.17981/ingecuc.12.2.2016.03
}

Artículo de Revisión Bibliográfica - Fecha de Recepción: 29 de Julio de 2015, Fecha de Aceptación: 7 de Abril de 2016

\author{
Oscar Jardey Suárez \\ Universidad Distrital Francisco José de Caldas, Universidad Autónoma de Colombia \\ Bogotá D.C. - Colombia. oscar.jardey.suarez@gmail.com
}

Cómo citar este artículo:

O. Suárez, "Aproximación al Origen de la Noción de Objeto de Aprendizaje: Revisión Histórico - Bibliográfica", INGE CUC, vol. 12, No.2, pp. 26-40, 2016. DOI: http://dx.doi.org/10.17981/ingecuc.12.2.2016.03

\begin{abstract}
Resumen- El objetivo de este artículo es aproximarse a la comprensión de la noción de Objeto de Aprendizaje (OA) desde una mirada histórica, en la que se evidencia que esta noción es derivada de la evolución del paradigma de la programación orientada a objetos; desde allí reflexionar las consecuencias de esta en el campo de la educación. El estudio es bibliográfico, recurre a fuentes primarias y secundarias para la construcción de los argumentos que se exponen. Para la organización se tuvo en cuenta dos hechos trascendentes: la noción de clases y subclases y la propuesta de OA. Las conclusiones de este trabajo señalan que la idea de OA no es espontánea, sino que es consecuencia de todo el desarrollo de la ingeniería, alrededor de los conceptos de clases y subclases, las cuales finalmente se transformaron en un paradigma, que propicia un contexto favorable, desde donde emerge como noción; adicionalmente se propone una mayor reflexión para la incorporación de esta noción en el campo de la educación.
\end{abstract}

Palabras clave- Clases y subclases; objeto de aprendizaje; paradigma orientado a objetos; recurso educativo abierto.

\begin{abstract}
The aim of this article is an approach to the understanding of the concept of Learning Object (LO) from a historical perspective, in which it is clear that this notion derived from the evolution of the paradigm of object-oriented programming; thence reflect the consequences of this on the education field. This work is part of the doctoral research entitled "Learning objects as cultural artifacts: conceptions of physics teachers working in the engineering faculty." The bibliographic study goes to primary and secondary sources for the construction of the arguments presented. For the organization two transcendent facts were took into account: the concept of classes and subclasses and the proposed LO. The conclusions of this study shown that the idea of LO is not spontaneous, but is the result of all engineering development around the classes and subclasses concepts, which eventually became into a paradigm that fosters a favorable context from which emerges as notion. Additionally further reflection for the incorporation of this concept in the field of education is proposed.
\end{abstract}

Keywords-Classes and subclasses; learning object; object-oriented paradigm; open educational resource. 


\section{INTRODUCCIÓN}

Este trabajo es resultado de una fase de la investigación doctoral titulada Objetos de aprendizaje como artefactos culturales: concepciones de los profesores de física que trabajan en la Facultad de Ingeniería, en la que se pretende comprender, desde el punto de vista histórico y bibliográfico, la noción de OA, así como a interpretar el desarrollo de esta noción y su posible incorporación en la educación, atendiendo la epistemología de las ciencia o de cada área de conocimiento.

La pregunta que direcciona este trabajo y que aporta a la investigación planteada es: ¿Cuál es el origen de la noción de OA? Esta aproximación intenta ser diacrónica, es decir, que se ocupa de develar las condiciones históricas que se dieron en el tiempo y que propiciaron un contexto fértil en el que emergió la idea de OA y tuvo una aceptación amplia para su desarrollo.

Los estados del arte, en relación con los OA, inician su desarrollo presentando el origen de estos como espontánea, como algo fortuito de una actividad visionaria y futurista; haciendo una analogía, pero guardando la distancia, la arquebiosis considera que en la fruta (como la guayaba) se genera de manera espontánea la mosca de la fruta ("Drosophila melanogaster"), hecho que perduró durante muchos años en las concepciones de sentido común y científicas; luego de ser refutada, de buscar y encontrar explicación a estos hechos se generó una revolución que ha implicado saltos de la humanidad en diversos sentidos, como el caso de la teoría de la evolución (que no será objeto de discusión en este artículo); luego entrar a considerar las razones del origen de la idea de OA, reiterando guardar las distancias, puede llevar a interpretar y comprender por qué el mayor desarrollo de los OA se ha dado desde el punto de vista técnico y tecnológico.

Estudiar los hechos ingenieriles trascendentales que transformaron la programación de computadores que dieron origen a la programación orientada a objetos, que tuvieron algunos orígenes e impacto económico, los hechos derivados, que han propiciado el desarrollo de la programación de computadores o la transposición a otras áreas de la ingeniería que llevó a transformarse en un paradigma, permite entender cuál fue el contexto en el que emergió la noción de OA y su amplio desarrollo en esta área.

La mirada a los OA, en el contexto y desarrollo de las Tecnologías de la Información y la Comunicación (TIC), ha llevado a pensar a los sec- tores gubernamentales que esta es una noción que aporta positivamente al sector educativo; hecho que se evidencia en las políticas nacionales de países como Colombia y Chile, que lo divulgan a través de sus portales con programas, materiales, concursos, etc. así como la apuesta de comunidades académicas conformadas y dedicadas a desarrollar la noción de OA, junto con sus posibles interacciones con otras áreas de conocimiento o desarrollo corporativo, tal como la Conferencia Latinoamericana de Objetos de Aprendizaje (LACLO).

En el contexto internacional, la Unesco en 2002 realizó el foro de recursos educativos de libre acceso, como una actividad frente al desarrollo de multiplicidad de materiales que circulan en la web de manera libre con licencia de dominio público o con licencia que permite su adaptación, uso o distribución; esta noción tiene similares atributos que los $\mathrm{OA}$, pero su principal propósito es aportar en mejorar "la calidad" del sector educativo; desde entonces esta organización ha continuado promoviendo el desarrollo e incorporación de esta noción en políticas nacionales; por ejemplo, en Colombia, el Ministerio de Educación ha decidido denominarlos Recursos Educativos Digitales Abiertos, que en su generalidad son iguales, pero se limitan a distribución a través de medios digitales.

\section{Metodología}

El trabajo tiene un enfoque de tipo bibliográfico [1]; el objetivo en esta fase de la investigación, es comprender el contexto histórico y desarrollo de donde proviene la noción de Objeto de Aprendizaje, de forma que permita entender los estados actuales de apropiación de esta noción, así como plantear algunas reflexiones en el campo de la educación que posibiliten promover su incorporación.

En relación con el proceso metodológico, es un trabajo cualitativo y está apoyado en fuentes bibliográficas $[1,2]$, mediante las cuales se busca indagar en fuentes primarias [3] y recurrir a fuentes secundarias con el propósito de que contribuyan a la comprensión y entendimiento del desarrollo y el contexto histórico de la ingeniería, en particular el antes, el hecho histórico y el después, de la que hizo emerger la programación orientada a objetos.

La organización de la información se hizo fundamentalmente en Ms Excel ${ }^{\circledR}$, Endnote ${ }^{\circledR}$ y Atlas ti ${ }^{\circledR}$, lo cual facilitó tanto la catalogación a partir de los metadatos, la organización, la comprensión de la misma, la estructuración y demás actividades. 
Tabla 1. Categorías para la organización de materiales

\begin{tabular}{|c|c|}
\hline CATEGORÍA & DESCRIPCIÓN \\
\hline$A \tilde{n} o$ & Año de la publicación. \\
\hline $\begin{array}{l}\text { Datos de la } \\
\text { publicación }\end{array}$ & $\begin{array}{l}\text { Se incluyen los metadatos de la } \\
\text { publicación, en los que se resalta si es } \\
\text { revista, memorias de congreso, libro, } \\
\text { manual, etc. }\end{array}$ \\
\hline Autores & Se escribe la cita en formato APA. \\
\hline Titulo & $\begin{array}{l}\text { Se digita el título del texto en su idioma } \\
\text { de origen y en español. }\end{array}$ \\
\hline $\begin{array}{l}\text { Palabras } \\
\text { Claves }\end{array}$ & Se escriben las palabras clave en español. \\
\hline $\begin{array}{l}\text { Resumen o } \\
\text { Abstract }\end{array}$ & $\begin{array}{l}\text { Se copia o hace el resumen del texto en el } \\
\text { idioma de origen y español. }\end{array}$ \\
\hline $\begin{array}{l}\text { Documento } \\
\text { primario }\end{array}$ & $\begin{array}{l}\text { Se especifica si el documento es una } \\
\text { fuente primaria o secundaria, desde el } \\
\text { punto de vista histórico. }\end{array}$ \\
\hline $\begin{array}{l}\text { Dirección } \\
\text { electrónica }\end{array}$ & $\begin{array}{l}\text { Si el documento tiene dirección y está } \\
\text { en la Internet, se escribe la dirección } \\
\text { electrónica de origen. }\end{array}$ \\
\hline
\end{tabular}

Fuente: Autor.

En Ms Excel ${ }^{\circledR}$ se construyó una tabla con categorías que permitieran clasificar y gestionar el material que aportaría al estudio (tabla 1); Atlas ti ${ }^{\circledR}$ se usó para enlazar los documentos y realizar la lectura de los mismos haciendo los "memos", categorizando los aportes; esto permitió organizar e identificar el hecho que se podría considerar como punto de inflexión en el desarrollo de la programación de computadoras; Endnote ${ }^{\circledR}$ contribuyó significativamente en la clasificación y escritura de los documentos.

De acuerdo con Kragh [3], las fuentes consideradas primarias se clasifican como institucionales confidenciales, entendidas como las memorias de congresos que se consideran como el hecho más relevante, luego se buscaron materiales que daban cuenta directa del desarrollo de la programación de compañías "líderes" en el desarrollo científico, como IBM, por ejemplo.

Este trabajo se aproxima a ser histórico, en la medida en que se basa en documentos originales [3] para interpretar e identificar los hechos que dieron lugar al desarrollo de la programación de computadores en lenguajes basados en objetos; sin embargo, la misma limitante de acceder a variedad de documentos de este tipo llevó al autor a retomar fuentes secundarias oficiales (escritas por otros autores), en las que se reconstruyen hechos históricos pertinentes, los cuales contribuyeron en la construcción de los argumentos que se exponen a lo largo del artículo.
Algunos elementos limitantes del enfoque metodológico que es necesario mencionar en el trabajo refieren a que el documento puede navegar entre el anacronismo y el diacronismo, como muchos textos científicos de orden histórico; sin embargo, la intención en su construcción fue la de ser objetivo, aunque puede presentarse incertidumbre al momento de la reconstrucción e identificación de los hechos históricos; es más, la relación de la noción de OA y su hipotética relación con el paradigma orientado a objetos se plantea a partir de los hechos que actualmente se identifican, referidos a la amplia aceptación y desarrollo de la temática principalmente en el contexto de la ingeniería, así como a la mutación de la noción de Objeto en la programación a otros contextos, como el de diseño de sistemas.

\section{III.RESULTADOS}

Los resultados se dividen en cuatro partes, las tres primeras orientadas a comprender el origen de la idea de OA en el contexto del desarrollo de la programación de computadores, la cuarta a evidenciar el desarrollo de la noción de OA. En esa línea de tiempo se identifica la propuesta de Dahl y Nygaard [4] como la que inicia este proceso, que trae como consecuencia la construcción del paradigma orientado a objetos, luego este hecho es el primer punto crítico elegido. En tal sentido, esta sección cuenta el antes, la propuesta y el después de este punto, hasta que en 1992 emergió el segundo punto crítico, en el que Hodigns, ejecutivo de AutoDesk ${ }^{\circledR}$, propone la idea de Objeto de Aprendizaje.

\section{A. Antes de la Programación orientada a Objetos (POO)}

Edmund Berckley, licenciado en matemáticas y lógica con experiencia en teoría de riesgo, en 1947, después de participar activamente en la Segunda Guerra Mundial, apoyó la fundación Association for Computing Machinery (ACM, https://www.acm.org/), con el propósito fundamental de divulgar los avances científicos del área de la computación; desde allí invitó a los militares para que divulgaran sus hallazgos, petición que fue respaldada por quienes hicieron parte de la ACM; de esta asociación se han derivado algunas comunidades y publicaciones vigentes a la fecha, como Journal Association for Computing Machinery: JACM (http://jacm.acm. org). En esta comunidad se gestaron varias ideas que a la postre se convirtieron en realidad en el área de la computación, tal es el caso del Paradigma Orientado a Objetos. 
Previamente, en el área de la computación, en 1941 , se presentó ante la comunidad científica alemana el computador Z3, cuya característica era resolver operaciones aritméticas utilizando binarios y apoyado en el concepto de "coma flotante", usada para escribir y operar números reales grandes y pequeños [5]; esta fue destruida en 1943 en uno de los ataques de los aliados a las instalaciones alemanas.

En los años siguientes, entre 1942 y 1945 , Konrad Zuse desarrolló el lenguaje de programación Plankalkül [6, 7], soportado en los conceptos de álgebra, el cual se frustró en su evolución y del que se registra una "primera" implementación de alto nivel en el año 2000, es decir, más de cincuenta años después de su propuesta inicial [8].

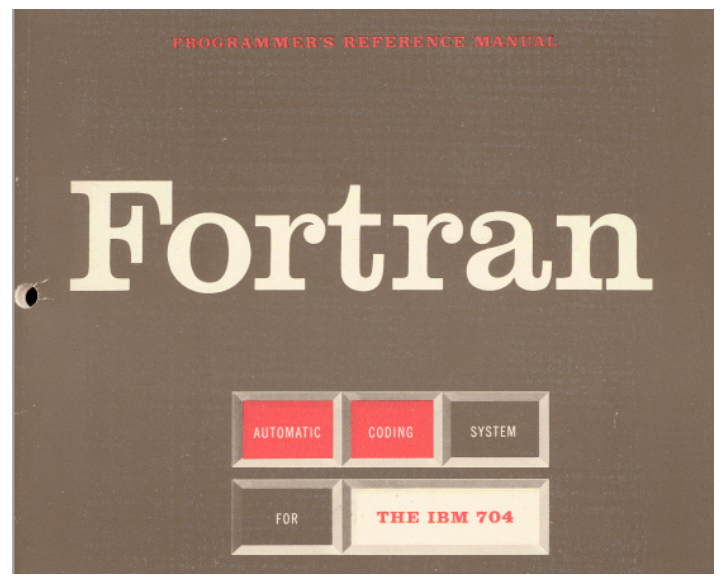

Fig. 1. Portada del manual original de FORTRAN publicado el 15 de octubre de 1956 por John Backus y otros para IBM Corporation Fuente: [9].

El matemático John von Neumann [10] retoma el trabajo de varios científicos, como el enigmático matemático Alan Turing (1912 - 1954), en el proyecto EDVAC (Electronic Discrete Variable Automatic Computer), la primera máquina orientada a resolver operaciones matemáticas en forma binaria, la cual fue diseñada y construida en la Universidad de Pensilvania en acuerdo con las Fuerzas Armadas de Estados Unidos [10]. En el reporte se identifica la complejidad de la máquina, así como el interés primario de tener un sistema interactuando para resolver operaciones matemáticas cada vez más complejas y en menor tiempo, pues la velocidad en los cálculos era y es importante para los fines armamentistas; el éxito de la máquina construida radica en que se constituyó en parte estructural de la arquitectura de computadores de diferente tamaño y cobertura.

Posteriormente, en 1946, se presentó el ENIAC (Electronic Numerical Integrator And Computer), que también fue construido en la
Universidad de Pensilvania en acuerdo con las Fuerzas Armadas de los Estados Unidos; de un tamaño considerable si la comparamos con las actuales. El ENIAC medía aproximadamente 170 metros cuadrados de área, que al compararlo con la actual Mac mini, cuya área es cerca de 400 centímetros cuadrados, indica que ocupaba más de 4250 veces el área de esta. La tecnología utilizada en la ENIAC, a diferencia del Z3 y del EDVAC, eran válvulas termoiónicas de base octal y realizaba un gran número de operaciones aritméticas, cerca de 5000 por segundo. Los problemas que resolvía no necesariamente eran de tipo militar; era bastante flexible pero tenía limitantes en entradas y salidas, así como fallas en su funcionamiento [11].

Ya en la década de los 50 emergieron diferentes formas de "programar" computadores, como consecuencia del interés investigativo y comercial; es el caso de FORmula TRANslation, FORTRAN (figura 1), caracterizado por un programa en línea con saltos GOTO [12]. El desarrollo de FORTRAN se extendió exitosamente hasta que en 1966 el American National Stantdar Institute (ANSI) lo estandarizó con el propósito de señalar la forma como se escriben e interpretan los programas desarrollados en este lenguaje, garantizando una mayor interoperabilidad de la variedad de los sistemas de procesamiento automático de datos [13]. Lo anterior resulta de especial interés, dado que en la categoría estándar establece la interconexión entre los diferentes desarrollos; este fue posteriormente revisado y ampliado [14].

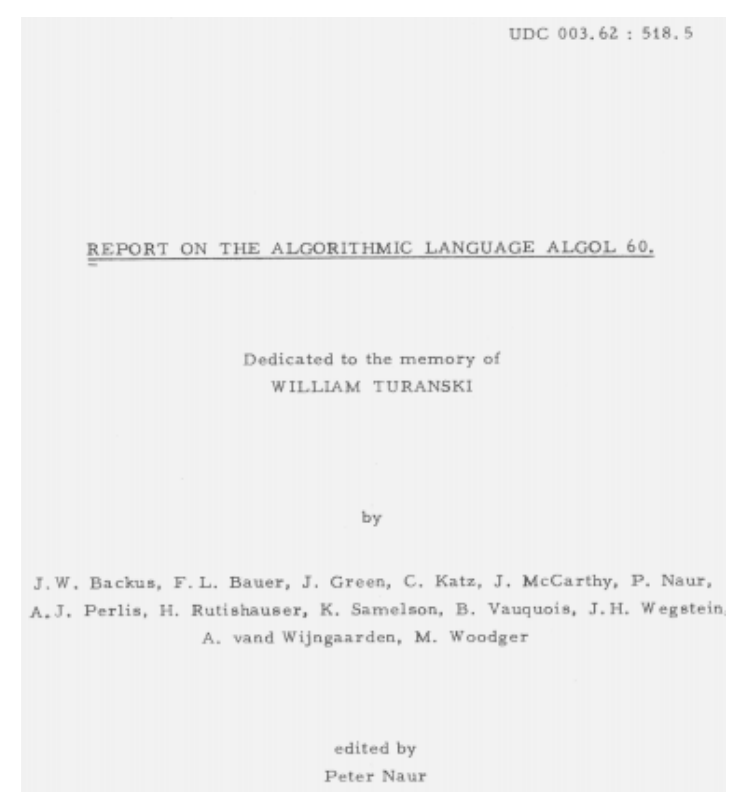

Fig. 2. Portada original del reporte de ALGOL 60 , editado por Peter Naur para la ACM Fuente: [9]. 
FORTRAN ha tenido gran trascendencia, y aún hoy día se encuentran desarrollos, fundamentalmente en las ciencias básicas como la física, debido a que los tiempos de compilación y ejecución de algoritmos es menor; aunado a esto, el gran acumulado de progresos que se han hecho en el área y que no se han migrado; ejemplo de esto es la tesis de Maestría que propone y valida, en FORTRAN, un algoritmo para el análisis del problema de dispersión de contaminantes en ríos [15]. Es interesante observar cómo, a pesar de la multiplicidad de herramientas actuales, este hace parte de los currículos, como en la Licenciatura en Física [16], en introducción a la física computacional [17], o para la asignatura de Introducción a los Métodos Numéricos en varias instituciones de educación superior.

De otro lado, el lenguaje ALGOL (figura 2), del que se tiene un primer reporte en 1958 en Zúrich, despertó un gran interés para lograr su desarrollo; después de una reunión informal en noviembre de 1958, Dahl y Nygaard se reunieron en febrero de 1959 en Copenhague (Dinamarca) para efecto de conformar un grupo orientado a desarrollar el hardware de manera "cooperativa".

Como dato interesante, en esa misma época LEGO ${ }^{\circledR}$, compañía danesa reconocida por elaborar juegos, tiene una gran transformación de su tecnología para el diseño de juegos que motivan la creatividad de los niños y niñas, impulsa los bloques para el diseño y construcción de estructuras, los cuales pueden reutilizarse para diversas figuras, brindándoles estabilidad. Este hecho resulta especial, dado que se produjo en un espacio y tiempo muy cercanos.

En reuniones preliminares a la conferencia de la ACM de 1960 se conformaron diversos comités para diferentes tareas, uno de los cuales corresponde a la programación. De la época un reporte tipo tutorial presenta una forma lineal de programación apoyado en funciones o procedimientos [18], de hecho aún hoy se conserva esta estructura; lo fundamental aquí es que las funciones o procedimientos son conceptualizadas como bloques, lo cual simplifica la escritura de los programas, pero no deja de ser complejo frente a los requerimientos logísticos de mantenimiento de software, lo cual se logra en buena medida cuando se reutiliza el código.

La compañía IBM en la década de los 60 disponía de una computadora a nivel de hardware denominada system/360 con fines de desarrollo y sobre todo comercial, en la que implementó un lenguaje parecido a ALGOL, denominado PL360, el cual tenía tres objetivos: 1) permitir el uso completo de los servicios proporcionados por el hardware 360,2) proporcionar mayor comodidad en la escritura y corrección de programas y 3) motivar a los usuarios finales para escribir con un estilo claro y comprensible sus propios desarrollos.

En este sentido, el lenguaje debió proveer los servicios suficientes para que controlen y supervisen la ejecución de los programas en el compilador. En este lenguaje está la función GOTO, muy novedosa en la época, que permite en tiempo de ejecución saltar entre bloques o segmentos del programa [19].

Es en este escenario que en 1952 Nygaard, inicia su trabajo de investigación de operaciones, cuya intención es conceptualizar el complejo mundo "real" en el marco del programa SIMULA, con la claridad de describir sistemas y llevarlos al lenguaje de programación [20]. En asociación con Dahl se reúnen en Oslo (Noruega) en el Congreso de la Federación Internacional de Procesamiento de la Información IFIP en 1962 y presentan el formal language proposal; idea que posteriormente daría lugar en 1967 al artículo que da la apertura al lenguaje y programación orientado a objetos y que es objeto de análisis en la siguiente sección.

\section{La propuesta o idea inicial de la programación orientada a objetos.}

SIMULA integra en su conceptualización clases, subclases (clases muy organizadas jerárquicamente), métodos (figura 4), Framework y administración de memoria automáticamente que, siendo un tanto anacrónico, hoy están presentes en los lenguajes de programación orientado a objetos como $\mathrm{C}++, \mathrm{C \#}$, Java [21], entre otros. Esto denota las características de los objetos, tales como la modularidad, reusabilidad, encapsulamiento, polimorfismo.

El mecanismo de conexión de SIMULA combina la plena seguridad con una mayor flexibilidad en un determinado gasto en la conveniencia y la eficiencia de tiempo de ejecución. El usuario se ve forzado, por la sintaxis de la instrucción de conexión, a determinar en tiempo de ejecución la clase de una estructura de datos de referencia antes de acceder a los posibles datos allí contenidos.

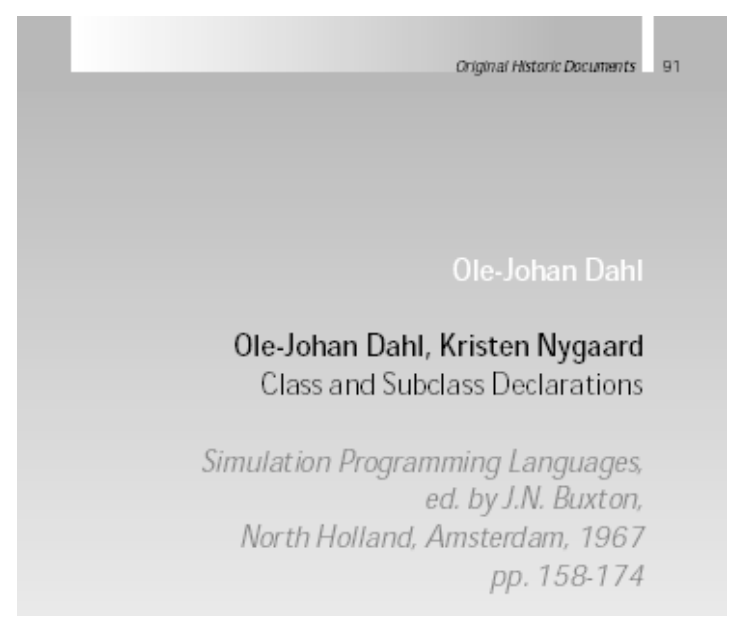

Fig. 3. Portada de las memorias de la publicación original hecha por Dahl y Nygaard [4] y que según los expertos marca la diferencia de dos generaciones en la programación Fuente: [9]. 
Dahl y Nygaard [4] (figura 3) amplían las ideas de autores anteriores, como Hore, en lo referido a la noción de clase, en un intento por superar las dificultades que había en ese entonces por la manipulación de estructura de datos y mantenimiento de software. El desarrollo de los autores muestra un enfoque con algunas convergencias y divergencias de trabajos anteriores; de hecho plantean que alcanza a considerarse como una extensión de ALGOL 60.

Dahl y Nygaard conceptualizan la idea de objeto como una instancia de una clase previamente definida o declarada. Los parámetros y cantidades declaradas localmente son los atributos de un objeto; estos pueden ser referenciados localmente dentro de la clase, pero también se puede acceder a ellos remotamente. Es preciso mencionar que lo expuesto en este párrafo refiere a la conceptualización semántica de la idea de objeto en el marco de la programación.

Una notación de prefijo se introduce para definir subclases organizadas en una estructura de árbol jerárquico; los miembros de una subclase son los llamados objetos.

De manera más específica, se conceptualizan los objetos como bloques de programación independientes (figura 5), que definen internamente sus variables, métodos y procedimientos cuyo alcance es local [4].

Dahl y Nygaard son presentados como los fundadores de la tecnología de objetos, como aquellos que dieron, desde el contexto que los rodeaba, el salto para concebir una forma diferente de programar, que en su momento resolvió muchísimos problemas de mantenimiento y desarrollo de programas [22] que tenían incidencia económica y de tiempo de respuesta.

Las ideas propuestas (clases y objetos, subclases, métodos virtuales entre otros) han perdurado en el tiempo, migrando a diferentes lenguajes de programación, tales como $\mathrm{C}++$ y Java, transponiéndose a otros ambientes de la ingeniería hasta convertirse en un paradigma.

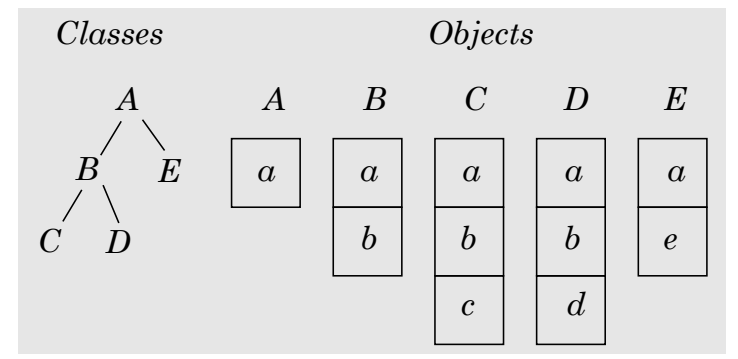

Fig. 4. Esquema tomado del artículo original que ilustra las clases A, B, C, D y E como objetos y una organización jerárquica con los respectivos componentes. Nótese que $\mathrm{B}, \mathrm{C}$, D y E son subclases de A y a su vez C y D son subclases de B Fuente: [9].

\section{El surgimiento del Paradigma orientado a Objetos}

Las compañías AT\&T y Bell desarrollaron lenguajes de programación C y Pascal (aproximadamente de 1969 a 1973), los cuales evolucionaron a $\mathrm{C}++$ a inicios de la década de los 80 , siendo estas expresiones propias de la programación OO.

Dennis Ritchie (1993) intenta aproximarse a narrar la historia evolutiva del lenguaje $\mathrm{C}$ desde la óptica de Bell, retomando el momento histórico de la compañía Bell en lo relacionado con sus investigaciones, así como la crisis con AT\&T, General y otras compañías; relata cómo fue el paso del lenguaje B al C y la forma como iban surgiendo las sentencias en la programación, así como la aparición del sistema operativo Unix.

No es parte de esta investigación ni del trabajo, pero se debe mencionar que existe una historia que no es objeto de estudio en este documento, pero que de manera rápida se comenta en el sentido de que AT\&T tuvo un monopolio de comunicaciones en Estados Unidos entre 1907 y 1982 junto a laboratorios Bell, lo cual da una idea de su injerencia en todos los desarrollos asociados a este, entre ellos los lenguajes de programación.

El lenguaje C, como proyecto de AT\&T y laboratorios Bell, se desarrolló ampliamente, al punto que fue evolucionando de $\mathrm{C}$ a $\mathrm{C}++$. En enero de 1984 Stroustrup promueve las cualidades de $\mathrm{C}++$, en particular la abstracción de datos [23]; esta característica es fundamental para efecto de generar una mayor dinámica en el manejo de la información y las variables, en lo referido a la escritura del programa y el manejo de la memoria. En ese mismo año, en septiembre, lanza un tutorial orientado exclusivamente a $\mathrm{C}++$, dejando de lado C; en este hace un desarrollo que hoy podría decirse típico: variables, tipos, operadores, entre otros [24]; finalizando el año 84 Strostrup escribe un texto en el que retoma los dos anteriores, pero enfocado al sistema operativo Unix, en el que resalta que $\mathrm{C}++$ es un lenguaje diseñado para desarrollar proyectos no triviales [25].

El desarrollo del lenguaje C, con todas sus características, evolucionó hasta convertirse en un estándar, tal como lo hizo FORTRAN, cuyo principal propósito es garantizar la interoperabilidad [26], lo cual quiere decir que un programa escrito en $\mathrm{C}$ puede ser compilado en cualquier plataforma, es decir, puede ser llevado a diferentes plataformas y allí leído, compilado y ejecutado, esta recomendación tuvo dos revisiones en el los años 90 у 99 .

De lo anterior es claro cómo la idea de Dahl y Nygaard es llevada y cristalizada ampliamente y en el tiempo en el ámbito de la programación, conservando las características inicialmente propuestas, poniendo especialmente énfasis en el 
desarrollo técnico y en la abstracción e interoperabilidad técnica garantizada, logrando migrar a productos muy conocidos actualmente.

En 1975 y 1976 nacen dos compañías, hoy emporios económicos, Microsoft y Apple, dirigidas al desarrollo de soluciones de computadores personales, las cuales utilizan la programación orientada a objetos en su desarrollo. En 1976 Apple I y en 1977 Apple II fueron los dos primeros ordenadores de Macintosh con algún éxito. En 1980 iniciaron el "proyecto Lisa", cuyo resultado fue el primer equipo Mac II (1984), que contemplaba ventanas superpuestas (basado en objetos), tecnología que Microsoft no podía utilizar. Sin embargo, en 1985 Microsoft lanzó Windows 1.0 con una diferencia sutil, las ventanas organizadas en Mosaico. Esta es la evidencia fuerte de los alcances que en su momento estaba teniendo el diseño y desarrollo de software con la idea de orientación a objetos, no solamente en la programación.

En otra línea de trabajo de la ingeniería, en 1981 la compañía Rational Machine, fundada por Paul Levi y Mike Devlin, tenía el propósito principal de propiciar prácticas modernas en Ingeniería de Software; el eje central de los servicios prestados por la compañía estaba en el modelamiento de sistemas desde la orientación a objetos, lo cual es evidencia de la migración de la noción propuesta por Dahl y Nygaard. Para 1994 la compañía estableció una alianza, a través de James Rumbaugh, con Grady Booch (Método Booch) e Ivar Jacobson, todos líderes y de alto nivel en el modelado y diseño orientado a objetos, quienes en conjunto, y con el apoyo de la comunidad académica, decidieron unificar el Lenguaje de Modelado en inglés Unifiqued Modeling Language (UML). Para ello establecieron un consorcio internacional UML en compañía de otras personas y entidades que condujeron a establecer la especificación aceptada y aprobada por la $\mathrm{Ob}$ ject Modeling Techique (OMT). Hoy la compañía Rational hace parte de IBM, que la adquirió en 2003 (por cerca de 2100 millones de dólares) y a través de su portal ofrece variedad de servicios cuyas características de diseño están basadas en la metodología orientada a objetos [27]. Todo lo anterior muestra de manera fehaciente la transposición de la idea de programación orientada a objetos al ámbito del diseño de sistemas de información y de software.

Esta amplia producción de conocimiento, conformación de comunidades, espacios de socialización y publicación permiten establecer que se ha consolidado un paradigma [28] entorno a la idea de "objeto" en el ámbito de la ingeniería, en particular de la Ingeniería de Software y de diseño de sistemas, lo cual se constituyó en campo fértil en la generación y desarrollo de ideas que se ajustarán a lo expuesto, como la noción de OA.

\section{La idea de Objeto de Aprendizaje OA}

Ya se mencionó la forma como se ha venido desarrollando la noción de programación orientada a objetos; de hecho se alcanza a revisar cómo esta migra a diferentes contextos hasta convertirse en lo que se denomina el Paradigma Orientado a Objetos; esto indica que en la cultura ingenieril hace parte del habitus [29] o esquemas, entendidos como algo preformado, como un patrón que se utiliza de manera inconsciente [30], dando origen al significado de la percepción, o como lo menciona Kerckhove [31], el interior de las personas (esquemas, habitus...) está presente en los productos y objetos externos, así como éstos muestran lo que está dentro, lo que se puede sintetizar en la frase "cuando tu interior está fuera y tu exterior está dentro".

Lo anterior para señalar que esa idea que concretan Dahl y Nyaargd en relación con las clases, subclases y objetos en el marco de la programación de computadoras, y que durante años ha tenido una fuerte evolución desde el punto de vista técnico, se constituyen en el cimiento y sistema de referencia cognitivo para que Hodgins [32, 33], en calidad de asesor futurista de compañías del sector de software, propusiera migrar de objeto en el marco de la programación de computadoras a objeto en el marco de la educación, denominándolo "objeto de aprendizaje”, apoyándose en un analogía ilustrativa como lo son las fichas y el desarrollo de LEGO ${ }^{\circledR}$.

Luego, la idea de diseñar y desarrollar OA nace en un ámbito técnico, tecnológico y, como consecuencia, proveniente de compañías dominadoras de un segmento del mercado del software; y es allí donde ha tenido un amplio desarrollo, pues se entiende la noción de objeto en diversos campos de la ingeniería; esta noción se resignifica o amplía su significado al contexto de la educación, inicialmente desde los contenidos o saberes, considerando que la educación es un campo complejo de la sociedad.

Lo anterior es el punto de partida de diversos estados del arte de los OA [34-37], en los que no se reflexiona alrededor de la comprensión del surgimiento de la noción de OA; es allí donde este documento hace uno de los principales aportes, pues la noción de OA no es una idea espontánea, es una idea que emerge de esquema o habitus de una comunidad ingenieril [38], que tiene una cultura académica [39] y una cimentación propia.

La noción de OA inicialmente es considerada como unidades digitales discretas cuyo principal propósito es que sean reutilizables [40-49], pero en la conceptualización se entraman dos capas: en la primera la cualidad de reusable y su intención clara de aprendizaje, y en la segunda lo interoperable, modular y granular [50]. 
La intención de un OA se refiere al objetivo al cual se espera aportar con el OA, enmarcado en un contexto educativo, cultural y disciplinar.

Lo reusable de un OA está en la medida que este puede ser utilizado en diferentes contextos, es decir, que tiene la cualidad de ser flexible, adaptable y escalable, atendiendo las limitaciones disciplinares, educativas y de contexto.

Lo granular en un OA se refiere a la precisión o fineza en el diseño y desarrollo, expresado en la cobertura de contenidos (por ejemplo, parte de una lección, una unidad temática, un curso).

Lo modular refiere la posibilidad de un OA de acoplarse para integrar uno o más ambientes de aprendizaje, conservando su independencia. Esta característica, como la granular, está descritas de manera eficiente en los metadatos.

Lo interoperable es una condición técnica que hace referencia a que puede ser usada en diversas plataformas, revisando estas características y comparándolas con las de "objeto" en la programación de computadores se alcanza a apreciar la similitud conceptual; si bien se considera el contexto educativo, es preciso señalar la particularidad que cada disciplina de conocimiento (biología, matemáticas, física, historia...) requiere ser considerada en relación con su didáctica, así como la forma como se configura el saber en la escuela [51, 52], del rol que juega el profesor en el liderazgo del desarrollo de la escuela, las implicaciones que tiene transponer el conocimiento desde la ciencia o disciplina de origen hasta convertirlo en el saber que circulará en la escuela[53], solo por mencionar algunos retos de cualquier aspecto que quiera trascender en la escuela o en los sistemas educativos.

Desde hace algún tiempo, las políticas educativas han hecho especial énfasis en algo denominado "calidad educativa", en la que la idea de OA encaja bien; así lo identifica al menos la Unesco, que desde 2002 y a la fecha ha venido trabajando la idea de Recurso Educativo Abierto. OA:

Considero como ampliación de la noción de

“... materiales de enseñanza, aprendizaje e investigación en cualquier soporte, digital o de otro tipo, que sean de dominio público ..., adaptación y redistribución por otros sin ninguna restricción o con restricciones limitadas..." [54].

Esta conceptualización está orientada a tener un principio de acceso a la información de manera libre y amplia, viendo esto como una oportunidad de llegar a lugares inhóspitos en el menor tiempo, como lo es la Internet o el ciberespacio, con acceso a la información y el conocimiento
[54, 55]. Esta consideración se enmarca en la idea de cobertura de la educación en sus diferentes metodologías y niveles, así como también de orden económico dependiente de las redes de comunicación, pero como tal no tienen ninguna consideración de orden filosófico, pedagógico o didáctico en el contexto de las disciplinas, es decir, se orientan hacia las especificidad y potencialidad de transportarse a diferentes distancias en corto tiempo de forma oportuna. Sin duda ha sido la discusión que se ha dado por diversos autores de los OA, el caso de Wiley [40, 56], quien estaba convencido del asunto y hoy, a través de su blog, se ha retractado de la noción de OA, dando el salto a la noción de REA [57], considerando que estos son libres de usar de manera perpetua para reutilizarlos, revisarlos, mezclarlos, redistribuirlos entre otras.

A la noción de REA, con apoyo institucional, se le ha dado matices, como el caso del Ministerio de Educación en Colombia, que lo refiere como Recursos Educativos Digitales Abiertos: REDA [37], respecto a lo cual la intencionalidad sigue siendo la misma, pero con sesgo únicamente en digitales.

Cuando se revisa en qué consiste la transformación de OA a REA se observa de fondo un criterio claro de libertad de uso del material, mediante la licencia "Creative Commons" o alguna similar, así como la inclusión de la posibilidad del plus de la investigación; estos hechos aportan en la idea de propender por una tecnología abierta, que apunte a una educación abierta, de bajo costo, con mejor acceso entre otros, tal como lo expone el mismo Wiley en su conferencia en TEDxNEYED en 2010 [58] y que comparte en su blog, lo que se adelanta en resolver una de las dificultades, el libre acceso a materiales.

Una de los mayores problemas en el acceso a los OA consistía en la "llave" (en ocasiones implicaba costo) necesario para acceder a los repositorios. Durante varias conferencias de LACLO se discutió el asunto. la Unesco con su idea de REA y el apoyo institucional de los gobiernos apunta conceptualmente a liberar los materiales educativos que pueden ser transportados por las redes de información; por ejemplo, LACLO estableció convenios de suscripción con Latin American Federation of Learning Object Repositories (FLOR) y Federación Mundial de Repositorios de Objetos de Aprendizaje (GLO$\mathrm{BE}$ ) con la idea de difundir los accesos libres a material educativo.

La pregunta que podría contribuir en el objetivo de este trabajo y que puede direccionar esta actividad puede ser ¿Qué ha pasado con la noción de OA entre su formulación inicial y su evolución o reformulación por parte de la Unesco? 


\section{Desarrollo de los objetos de aprendizaje $O A$}

Diversas instituciones han hecho esfuerzos para contribuir en el desarrollo de la idea de OA tal como Institute of Electrical and Electronics Engineers: IEEE[59], IMS Global Learning Consortium [60], Alliance of remote instructional authoring and distribution networks for Europe (ARIADNE) y Advanced Distributed Learning Network (ADL); junto a ellas se agruparon diferentes personas y compañías para trabajar en torno a la idea de OA. Específicamente, la IEEE configuró el grupo de trabajo denominado Learning Thechnology Standards Committee (LTSC), con un subgrupo denominado Learning Objects Metadata (LOM), que interactúo con ADL y generó en 2002 la norma Sharable Content Object Reference Model (SCORM) versión 1.2 [61], que es utilizada, entre otros, para catalogar los OA a partir de sus metadatos.

La norma Scorm 1.2 ha sido ampliamente utilizada para desarrollar variedad de productos que han sido certificados, tales como los sistemas de administrador de aprendizaje Pegasus LMS 5.9, Xyleme LMS 3.1, LMS System based on e-book v1.0 $\mathrm{u}$ otros productos de orden más específico como TM SIGAL v6, Health Stream Learning Center Diabetes Essentials Catalogue Courseware v 1.0, entre los más de trescientos productos [62].

Otros trabajos han tenido la idea de orientarse a unir o concatenar la noción de OA con conceptos existentes en otros campos disciplinares, y han generado intersecciones que tienden una red de conocimiento que soporta la idea de los OA. Por ejemplo, en una aproximación a una ontología de Objeto de Aprendizaje Yen y Wang [63], basados en las recomendaciones de la Association for Computing Machinery (ACM) y la Association for Information Systems (AIS) [64] y que posteriormente la retoma Topi y otros [65], ubican los OA como unidades atómicas de conocimiento interrelacionadas, compartibles y reutilizables, que pueden ser abordadas de manera dinámica con diferentes estrategias en ambientes e-Learning, creando formas de fácil adaptación.

Entre los eventos dedicados a la divulgación y promoción, así como a la generación de dinámicas propias de una comunidad académica de OA de habla hispana, están el Simposio Multidisciplinario Sobre el Diseño y Evaluación de Contenidos Educativos Digitales (SPEDECE) y la Conferencia Latinoamericana de Objetos de Aprendizaje: LACLO (Latin-American Community on Learning Objects), que han venido desarrollando la convocatoria y discusión año tras año, desde mediados de la década del 2000, en intentos por mantener una discusión de temas comunes; como dato adicional, estas comunidades han desarrollado al menos un evento en común.
El caso de LACLO, que se desarrolla desde 2006, nació gracias al liderazgo de Xavier Ochoa desde la Escuela Superior Politécnica del Litoral en Guayaquil (Ecuador) en compañía de otros profesores; LACLO fue creada con la intención de ser referente de desarrollo de esta área de conocimiento en la región; a la fecha ha cumplido tal propósito en varios países, como Brasil, Colombia, Ecuador, Chile entre otros, que expresamente han retomado algunas de sus ideas para promoverlas desde los entes institucionales, como los ministerios de Educación, así como en algunas publicaciones [37].

LACLO ha estado promoviendo diferentes subáreas relacionadas con el uso de las Tecnologías de la Información y la Comunicación (TIC), las cuales se han dinamizado a lo largo de la línea de tiempo. El tema que ha prevalecido es el de Objeto de Aprendizaje, el cual se convoca actualmente con el de Recursos Educativos Abiertos, alrededor de los cuales se han hecho concursos; otros temas recientes han sido el diseño y desarrollo de APP, relato de experiencias con el uso de las Tic, Web 2.0, métricas de evaluación de OA o REA, entre otros temas.

Entre los múltiples desarrollos, propuestas y proyectos reportados a través de artículos se encuentran diversas experiencias en el campo de la educación, en diferentes áreas que han intentado aproximarse al uso de los OA en las aulas en los diferentes niveles educativos, tal como para la enseñanza de la bibliotecología [66], el aprendizaje de la farmacología [67], la ingeniería de software[41], entre muchos otros proyectos en el ámbito de la experiencia educativa [68-72]; algunas iniciativas han estado dirigidas a crear referencias para el diseño, desarrollo, catalogación, adaptación y concreción exitosa de los OA [73], la metodología de los OA [74-78], otros se relacionan con la evaluación de los OA en su principal característica, la reusabilidad [79].

La reutilización como el plus de los OA la han revisado Ochoa y Duval [80], en un estudio basado en la Learnométrica, en el cual indican que el $20 \%$ de los OA presentes en los repositorios son reutilizados sin importar su tamaño ni granularidad. Sin embargo, los indicadores como el mencionado tienen una incertidumbre que los hace no muy confiables debido al origen diverso y heterogéneo de los datos, así como a las técnicas de captura y procesamiento de información [81].

Previamente López, Escalante y Sánchez [82] identificaron que la mayoría de los OA analizados, en un estudio hecho a Merlot, carecen de mecanismos apropiados para lograr una reutilización satisfactoria; conclusión que toman de los factores definidos, como: metadatos, independencia de la presentación de contenido, granularidad, interacción persona $\mathrm{OA}$, generalidad del 
lenguaje y elementos de la interfaz, organización de contenidos, uniformidad de la presentación.

Lo anterior, que se percibe actualmente, pone en discusión la esencia de "la reutilización" los $\mathrm{OA}$, es decir, si ésta si está logrando el alcance prometedor de la noción de OA.

De otro lado, algunas otras miradas optimistas señalan que el crecimiento de la web es una oportunidad de promover la reutilización y proponen los retos en su gestión; en particular se proponen la integración y gestión de OA provenientes de diversos repositorios, con el fin de ahorrar tiempo y subir estándares de calidad [83].

Algunos programas, además de las políticas gubernamentales, son señal de la influencia de esta noción; entre estos está, desde lo educativo, "Alter-nativa", cuyo objetivo se centra en la construcción de referentes curriculares con incorporación de la tecnología, para facultades de educación en matemáticas y ciencias para atender poblaciones en contexto de diversidad en Chile, Colombia, Nicaragua, Perú, España, Argentina, México y El Salvador. Centra su propuesta en los OA o en sus sinónimos Objeto Virtual de Aprendizaje (OVA), Objetos de Aprendizaje Digitales [84].

León [85] compiló la acción del grupo de investigación Grupo de Investigación Interdisciplinaria en Pedagogía de Lenguaje y las Matemáticas (GIIPLyM), en el proyecto "alternativa", en un texto en el que propone referentes curriculares para la formación de docentes en matemáticas, quienes a partir de escenarios naturales que incorporan los OA identifican sus potencialidades en la formación de profesores de matemáticas, atendiendo a la diversidad cultural.

Hasta aquí se ha identificado cómo emerge la noción de Objeto de Aprendizaje, la cual se concreta en la idea de clases y subclases de Dahl y Nygaard [4], no como una idea espontánea sino como consecuencia del esquema o habitus de la cultura ingenieril, desde la noción de clases y subclases, que finalmente evoluciona hasta llegar a ser un paradigma que se denomina "orientado a objetos"; este paradigma ha logrado importantes resultados en diversas áreas de la ingeniería.

las condiciones favorables que facilitaron emerger la idea de OA son, entre otras: a) un contexto histórico de desarrollo amplio de la Internet que conlleva la abundancia de información, b) cada vez más posibilidades de conectividad, c) las necesidad de cobertura del sistema educativo, d) necesidad y posibilidad de rápida difusión de la información, en formatos flexibles y adaptables.

La idea de OA que luego se transformó, o fue absorbida, en la noción de REA, recurriendo principalmente a temas de licenciamiento y costo, al menos así se infiere de la última conceptualización:
... cualquier recurso educativo (incluso mapas curriculares, materiales de curso, libros de estudio, streaming de videos, aplicaciones multimedia, podcasts y cualquier material que haya sido diseñado para la enseñanza y el aprendizaje) que esté plenamente disponible para ser usado por educadores y estudiantes, sin que haya necesidad de pagar regalías o derechos de licencia...[86].

La prevalencia y primacía de los referentes técnicos y tecnológicos en los $\mathrm{OA}$ es evidente, como se ha señalado en los diversos argumentos.

Por ejemplo, Sprock y sus colegas [78] hacen un estado del arte de las metodologías propuestas para el diseño, desarrollo, validación, implementación y catalogación de los $\mathrm{OA}$, en el que se identifican 8; varias provienen "explícitamente" de la ingeniería de software;en ellas se hacen adaptaciones para la inclusión de los aspectos didácticos o pedagógicos del OA; es el caso de la metodología ISDMELO (Instructional Systems Development Methodology based on e- Learning Objects), que se fundamenta en la metodología de diseño y desarrollo de software ADDIE (Análisis, Diseño, Desarrollo, Implementación y Evaluación).

ADDIE en su proceso considera como aportantes de información "el perfil del aprendiz", tal como se hace al diseñar un sistema de información con "el perfil de usuario"; esto de alguna manera es una transposición del esquema ingenieril de diseño de sistemas de información a material didáctico orientado, principalmente, desde los aspectos técnicos y tecnológicos.

\section{Consideraciones Finales y Conclusiones}

Este trabajo nació de la investigación doctoral, cuya necesidad es comprender de dónde emerge la idea de Objeto de Aprendizaje, que luego se transforma o evoluciona en Recursos Educativos Abiertos (REA). En este ejercicio se identifica que la noción de OA no es una idea espontánea, sino que es consecuencia de un saber que está apropiado en la cultura de la ingeniería de clase y subclase, que luego se transpone a la de Objeto y de la que se deriva un volumen significativo de trabajos en diversas áreas de la ingeniería.

El hecho de que la noción de objeto esté vinculada al habitus, esquema o concepciones de la cultura ingenieril hace que sea esta comunidad la "tierra fértil" para su cultivo y desarrollo; en tal sentido, se observa cómo los mayores desarrollos alrededor de la noción OA provienen de la ingeniería, tales como metodologías, métricas, minería de datos, patrones de desarrollo, entre otros. 
En 1967 los asuntos que llevaron a pensar una solución innovadora a los problemas de los sistemas fueron relacionados con su mantenimiento, el manejo de la información, los cuales se traducían en cifras económicas; es más, la finalidad de avanzar en este sentido era tener usuarios con sus propios desarrollos y que ellos hicieran el mantenimiento a sus programas de manera menos complicada, y por eso se da el salto en el concepto de la programación; de forma anacrónica, en 1994, con la Internet, se provoca abundante información, lo cual genera problemas relacionados con el almacenamiento, transmisión y organización entre otros.

Para 1994 la Internet movilizó grandes volúmenes de información a una población en crecimiento; esto permitió visionar nuevos negocios para renglones ya existentes y crear otros. La educación, siendo un propósito social y un renglón económico (Colombia dedica el 16\% de su presupuesto, Estados Unidos el $12 \%$ ), fue visto por compañías multinacionales de software que dedicaron esfuerzos tendientes a configurar las características del negocio. Hodgins, contratado por multinacionales, propone la mencionada idea de los OA, con las consecuencias para nuevas y existentes comunidades académicas y para las existentes áreas de la educación.

La IEEE se suma a los esfuerzos por normatizar el diseño, desarrollo y catalogación de los $\mathrm{OA}$; los gobiernos en general se han preocupado por incrementar la densidad de equipos en las escuelas, la conectividad y los accesos a la Internet y demás aspectos de dotación en las escuelas; junto a esto también se han propuesto programas (como Computadores Para Educar, en Colombia) tendientes a favorecer la incorporación efectiva de las TIC en el medio educativo, lo cual aún no reporta resultados generalizables referidos a mejores aprendizajes por parte de estudiantes.

La expansión de las TIC en las escuelas, así como el acceso a contenidos en las redes de información, junto con las diversas formas de interacción social, se convierten en un reto y problema para la escuela y en general el sistema educativo; el diseño, desarrollo y evaluación de contenido (OA) desde y hacia las escuelas con niveles de calidad y pertinencia en términos culturales, nivel educativo y área de conocimiento, de otro lado, la divulgación de OA exitosos en contextos específicos o diversos de forma que se reutilicen, es una de las actividades aún por hacer.

Cada área de conocimiento tiene su propia epistemología, los conocimientos provenientes de cada una de estas son sometidos a una transformación de orden didáctico, hasta convertirse en un saber que circula en la escuela [51, 52]; esta labor se deja al campo de conocimiento didáctico general y específico de cada área.

La didáctica general y específica de cada área, en alguna medida, es una de las convocadas a liderar el cómo, el momento, el porqué y el para qué de la incorporación de las TIC, en particular los OA en los diversos ambientes de aprendizaje, dado que los docentes tienen el nivel directo para usar los OA en la escuela.

El origen y consolidación de los $\mathrm{OA}$ ha sido principalmente en el contexto técnico y tecnológico; esta idea se ha afianzado con múltiples desarrollos en la ingeniería; por ejemplo, eXeLearning es resultado de un proyecto iniciado en el Gobierno de Nueva Zelanda, que posteriormente retomó el Instituto de Tecnologías Educativas de España, al que se han vinculado compañías y personas naturales en su desarrollo; inicialmente se pensó desde los OA, pero se ha ampliado a diversos contenidos y condiciones técnicas, aproximándose a una solución técnica y tecnológica funcional, con posibilidad de interoperabilidad, orientado a la web, con licencia de libre uso; esta solución se orienta al contexto educativo, así como Clic y Jclic, que son soluciones multiplataforma en la web, disponibles para que los docentes y las escuelas lo utilicen en sus ambientes de aprendizaje.

El alcance de los $\mathrm{OA}$ se ha llevado al nivel de los Ambientes de Aprendizaje (AA), lo cual considero es una equivocación. Los AA son espacios, presenciales o no presenciales, que se definen mediante una intención, bajo las dinámicas de los procesos educativos en las que las acciones, expectativas y experiencias de los involucrados, así como actitudes, condiciones sociales, científicas y culturales y la infraestructura [87], son consideradas; en tanto que los OA pueden considerarse potencialmente como una mediación que puede hacer parte del ambiente de aprendizaje.

Asumir los OA como mediación. La mediación entre el conocimiento y su transposición al saber en la escuela se produce de diversas formas, y se relaciona directamente con la epistemología de cada área de conocimiento; considerar los OA como una mediación podría potencialmente contribuir a dar el salto a que una solución técnica y tecnológica aporte en el diseño de AA en la escuela. En este sentido se espera que los docentes, en los diferentes niveles de los sistemas educativos, empiecen a valorar cómo un OA podría mediar entre el conocimiento proveniente de las ciencias o áreas de estudio y el saber que circula en la escuela.

La producción de $O A$ debe enmarcarse en la epistemología de cada ciencia o área de conocimiento. Los OA en su origen y desarrollo han estado desprovistos de una epistemología de la 
ciencia o área destino; dicho de otra forma, los OA se originaron y desarrollaron en el contexto propio de la ingeniería con sólidos fundamentos técnicos y tecnológicos; en su producción deben ligarse profundamente con la epistemología de cada ciencia o área de conocimiento, de forma que los OA se aniden a las creencias de estas, potencializando un mejor plus al momento de ser utilizado en algún Ambiente de Aprendizaje.

Finalmente, los OA son, desde la ingeniería, una solución para la gestión y organización de la información; sin embargo, han sido muy criticados porque su principal idea, la reutilización, no ha tenido el impacto esperado, así como su contribución a la obtención de mejores resultados de aprendizaje, es decir, incrementar las buenas calificaciones en un mayor número de estudiantes. Esta abstracción se ha dejado para que sea el campo de la educación el que los incorpore; sin embargo, las iniciativas deben incluir, amplia y concienzudamente, los actores que en ella intervienen, principalmente a los docentes, quienes en últimas lideran y direccionan su incorporación en los espacios formales en las diferentes situaciones o ambientes; coincidiendo parcialmente, en una transposición, con Bachelard [88] se debe estudiar las concepciones o esquemas de los profesores, de forma que se identifiquen los posibles obstáculos que pueden impedir o dificultar la incorporación de estas nociones en las prácticas pedagógicas. Lo anterior no contradice el hecho de que el aprendizaje es personal, solamente que éstas nociones, OA y REA, aportan otras mediaciones que podrían estar potencialmente próximas a la actual cultura estudiantil.

\section{Agradecimientos}

Se da especial agradecimiento al Centro de Investigación y Desarrollo Científico (CIDC) de la Universidad Distrital, que apoya económicamente el proyecto doctoral; a mis hijos, quienes me han apoyado con el sacrificio de nuestro tiempo en familia. Dedico de manera especial este artículo a la Dra. Sonia Herminia Roa Trujillo, quien aportó a las decisiones que transformaron y dinamizaron un momento muy especial de mi vida.

\section{VI.FINANCIACIÓN}

Artículo de investigación científica derivado del proyecto de investigación "Objetos de aprendizaje como artefactos culturales: concepciones de los profesores de física que trabajan en la Facultad de Ingeniería". Financiado por la Universidad Distrital Francisco José de Caldas, Código 4-601-373-13. Fecha de inicio: 11 de julio de 2013. Fecha de finalización: julio de 2016.

\section{REFERENCIAS}

[1] A. M. Rodríguez and M. A. Moya, La investigación en la era de la información. México: Trillas, 2008.

[2] A. Quintana, "Metodología de Investigación Científica Cualitativa", in A. Quintana and W. Montgomery Eds., Psicologia: tópicos de Actualidad. Lima, Perú: Escuela Académico Profesional - Facultad de Psicología, 2006, pp,. 47-85.

[3] H. Krag, Introducción a la historia de la ciencia. BarcelonaEspaña: Crítica, 1989.

[4] O.-J. Dahl and K. Nygaar, "Class an Subclass Declarations", presented at the Simulation Programming Languajes, Amsterdam, North Holland, 1967.

[5] IEEE, "Standard for Floating-Point Arithmetic. Sustituyó la recomendación IEEE_ 754- 1985", 2008.

[6] W. K. Gioli, "Konrad Zuse's Plankalkül: the first high-level, "non von Neumann" programming language," Annals of the History of Computing, IEEE, vol. $19, \mathrm{n}^{\circ} 2$, pp. $17-24,1997$.

[7] F. Bauer and H. Wössner, "The "Plankalkül" of Konrad Zuse: A Forerunner of Today's Programming Languages", Magazine Communications of the ACM, vol. 15, $\mathrm{n}^{\circ} 7$, pp. $678-685,1972$.

[8] R. Rojas, R. Rojas, C. Göktekin, G. Friedl, M. Krüger, F. U. Berlin, F. U. Berlin, G. Friedl, M. Krüger, and F. U. Berlin, "Plankalkül: The First High-Level Programming Language and its Implementation," 2000.

[9] K. Kleine, Historic Documents in Computer Science, December 2013. Available: http://www.eah-jena.de/ kleine/history/

[10] J. von Neumann and John, "First draft of a report on the EDVAC," IEEE Ann. Hist. Comput., vol. 15, no. 4, pp. 27-75, 1993.

[11] J. Presper and Mauchly, "Patente $\mathrm{n}^{\circ} 3.120 .606$. Electronic Numerical Integrator and Computer June 26 de 1947", United States Patent Office Philadelphia - Estados Unidos de América, 1964.

[12] Bakus et al., The Fortran Automatic Coding System For The IBM 704 EDPM. Programmer's Reference Manual. New York, Estados Unidos: IBM, 1956.

[13] A. N. S. I. ANSI, "American National Standard Fortran”. New York, Estados Unidos de América: ANSI, 1966.

[14] A. N. S. I. ANSI, Programming Language FORTRAN X3.9-1978 Revision of ANSI X3.9-1966. New York, Estados Unidos de América: ANSI, 1978.

[15] C. H. Galeano-Urueña, "Técnicas de solución numérica de la ecuación de Difusión-Advección-Reacción para el estudio de dispersión de contaminantes", Facultad de ciencias Universidad Nacional de Colombia, Bogotá, Colombia, 2009.

[16] L. E. Física, Programación y lenguaje Fortran, 2015. [Online]. Available: http://licfis.fisica.uson. $\mathrm{mx} /$ programas/bas/9152.pdf

[17] D. D. Física, Statistical and Computational Physics Laboratory - Fisica Computacional - Introduccion a la Fisica Computacional, 2015. Available: http://lafec.df.uba.ar/fiscomp.html 
[18] P. Naur et al., "Report on the Algorithmic Language ALGOL 60". Paris, Francia: Association for Computing Machinery , 1960.

[19] U. STANFORD, PL360 Manual de Reference. California, Estados Unido de América: Stanford University, 1975 .

[20] J. Sklenar, Introduction to oop in simula, 1997. [online] Available: http://staff.um.edu.mt/jskl1/talk. html

[21] H. Deite and Deitel, C/C++ y JAVA. Como programar. México DF, México: Pearson Educación, 2004.

[22] B. Meyer, "In memory of Ole-Johan Dahl and Kristen Nygaard," in Journal of object technology, vol. 1, $\mathrm{n}^{\circ}$ 4, pp. 7-15. 2002.

[23] B. Stroustrup, Data Abstracción in C. New Jersey, Estados Unidos de América: Murray Hill, AT\&T Bell Laboratories, 1984.

[24] B. Stroustrup, Computing Science Thecnical Report No 113 A C++ Tutorial. Nueva Jersey, Estados Unidos de América: Murray Hill, AT\&T Bell Laboratories, 1984.

[25] B. Stroustrup, "Computing Science Technical Report No 108, The C++ Programming Languaje Reference Manual”. Nueva Jersey, Estados Unidos de América: Murray Hill, At\&TBell Laboratories, 1984.

[26] A. N. S. I. ANSI, American National Standard for Information System Programming Language C X3. 159. New York, Estados Unidos de América: ANSI, 1989.

[27] I. B. M. CORPORATION, IBM, 2016.

[28] T. Kuhn, La estructura de las revoluciones científicas. México: Fondo de Cultura Económica, 1971.

[29] A. Moreno-Durán and J. Ramírez, Introducción elemental a Pierre Bordieu. Bogotá, Colombia: Panamericana formas e impresos, 2006.

[30] A. Rosa and I. Bresó, "F. C. Bartlett, una antropología desde la psicología experimental", AIBR. Revista de Antropología Iberoamericana, núm. Esp., noviembre-diciembre, pp. 1 - 29, 2005.

[31] D. Kerckhove, La Piel de la Cultura - Investigación la nueva realidad electrónica. Barcelona, España: Gedisa, 1999.

[32] P. Taylor-Northrup, Learning Objects for Instruction: Design and Evaluation: Design and Evaluation. United States of American and United Kingdom: Idea Group Inc., 2007.

[33] W. Hodgins, H. Wayne Hodgins, 2016.[Online]. Available: https://http://www.computer.org/web/ awards/karlsson-wayne-hodgins

[34] G. Astudillo, "Análisis del estado del arte de los objetos de aprendizaje. Revisión de su definición y sus posibilidades", Facultad de Informática Universidad Nacional de La Plata, Buenos Aires, Argentina, 2011.

[35] M. Callejas-Cuervo, E. J. Hernández-Niño, and J. N. Pinzón-Villamil, "Objetos de aprendizaje, un estado del arte”, Entramado, vol. 7, n 1, pp. 176 - 189, 2011.

[36] J. Corona-Flores and B. González-Becerra, "Objetos de aprendizaje: Una Investigación Bibliográfica y Compilación”, RED. Revista de Educación a Distancia, vol. 34, pp. 1-42, 2012.
[37] MEN, Recursos Educativos Digitales Abiertos - Colombia. Bogotá, D.C., Colombia: Ministerio de Educación Nacional, 2012.

[38] B. Milicic, V. Sanjosé, G. Utges, and B. Salinas, "La cultura académica como condicionante del pensamiento y la acción de los profesores universitarios de física”, Investigações em Ensino de Ciências, vol. 12, pp.263-284, 2007.

[39] T. Becher, Tribus y territorios académicos, la indagación intelectual y las culturas de las disciplinas Barcelona,España: Gedisa, 1989.

[40] D. Wiley, "Learning Object Design And Sequencing Theory", Brigham Young University, Faculty Philosophy, 2000.

[41] I. Douglas, "Instruccional Desing Based on Reusa ble Learning Objects: Applyng Lessons of ObjectOriented software engeneering to learning system desing", in 31 ASEE/iEEE Frontiers in Education Conference, Reno, Estados Unidos de América, 2001.

[42] A. Altun and P. Askar, "From Blocks to Granules: An Alternative Approach to designing learning ob jects", in IEEE Computer Society - Advanced Learning Technologies, 2008. ICALT' '08. Eighth IEEE International Conference on, Santander, Cantabria, España, 2008.

[43] P. Mohan, S. Bucarey, and B. Daniewl, "Employing Object-Oriented Design Principles in the Design of Learning Objects in a Software Engineering Course", in IEEE Computer Society. Proceedings of the Sixth International Conference on Advanced Learning Technologies (ICALT'06), Kerdrake, Paises Bajos, 2006.

[44] C. Brooks, "Learning Objects on the web Semantic Web", in $3^{\text {rd }}$ International Conference on Advance Learning Technologies (ICALT'03), Atenas, Grecia, 2003.

[45] B. Defude and R. Farhat, "A Framework to Desing Quality-Based Learning Objects", in Fifth IEEE International Conference on Advance Learning Tech nologies (ICALT'05), Kaohsiung, Taiwan, 2005, pp. $1-5$.

[46] R. McGreal, "Learning Objects and Metadata", presented at the International Wrokshop on Technology for Education (T4E). IEEE, Bangalore, India, 2009.

[47] L. Santacruz, I. Aedo, and C. Delgado, "Designing Le@rning Objects with the ELO-Tool”, in $3^{\text {rd }} I E E E$ International Conference on Advanced Learning Technologies (ICALT'03), Atenas, Grecia, 2003.

[48] O. Suárez, L. Suárez, C. Sánchez, and M. Castilla, "Metodología para el diseño, desarrollo y evaluación de Objetos Virtuales de Aprendizaje", in Universidad Virtual 2008, La Habana, Cuba, 2008.

[49] S. H. Pitkanen, F. Joensuu-Univ, and P. Silander, "Criteria for Pedagogical Reusability of Learning Objects Enabling”, in IEEE International Conferen ce on Advanced Learning Technologies (ICALT'04), Beijing, China, 2004.

[50] O. Suárez, "Ambientes virtuales de aprendizaje basados en tecnología orientada a objetos", Facultad de Ingeniería Universidad Distrital Francisco José de Caldas, 2003. 
[51] L. Zambrano, Pedagogía, Didáctica y Saber. Bogotá: Editorial Magisterio, 2005.

[52] A. Zambrano Leal, "Las ciencias de la educación y didáctica: hermenéutica de una relación culturalmente específica," Educere, vol. 10, no. 35, pp. 593599, 2006

[53] Y. Chevallard, La transposicón didáctica del saber sabio al saber enseñado: Grupo editor AIQUE, 1998.

[54] Unesco, Declaración de París de 2012 sobre los REA, 2012. [Online]. Available: http://www.unesco. org/new/fileadmin/MULTIMEDIA/HQ/CI/CI/pdf/ Events/Spanish_Paris_OER_Declaration.pdf

[55] Unesco, Comunicación e Información - Recursos Educativos Abiertos,[Online]. Available: http://www. unesco.org/new/es/communication-and-information/ access-to-knowledge/open-educational-resources/

[56] D. Wiley, The instructional use of learning objects, 1st ed. Bloomington, Ind.: Agency for Instructional Technology-Association for Educational Communications \& Technology, 2002.

[57] D. Wiley, Of Sunlight, OER, and Lumen, 2016.

[58] D. WILEY, Open Education and the Future, 2010. [Online]. Available: https://http://www.youtube. com/watch?v=Rb0syrgsH6M\&feature=youtu.be

[59] IEEE, IMS Meta-data Best Practice Guide for IEEE 1484.12.1-2002 Standard for Learning Object Metadata, [Online]. Available: http://www.imsglobal.org/ metadata/mdv1p3pd/imsmd_bestv1p3pd.html

[60] I. G. L. Consortium, IMS GLOBAL Learning Consortium Better Learning From Better Learning Technology, 2016. [Online]. Available: https://http:// www.imsglobal.org

[61] P. DODDS, Sharable Content Object Reference Model SCORM Version 1.2 Advanced Distributed Learning, 2001.

[62] A. D. Learning, SCORM Certified Products / SCORM Adopters, [Online]. Available: http://www. adlnet.org/scorm/scorm-version-1-2.html

[63] M. Lee, D. Yen, and T. Wang, "Java Learning Object Ontology”, in Fifth IEEE International Conference on Advanced Learning Technologies (ICALT'05), Kaohsiung, Taiwan, 2005.

[64] J. Croos, E. Roberts, and Y Otros, "Computing Curricula 2001 Computer Science", The Joint Task Force on Computing Curricula IEEE Computer Society Association for Computing Machinery, 2001.

[65] H. Topi et al., "Curriculum Guidelines for Undergraduate Degree Programs in Information Systems", Association for Computing Machinery (ACM)- Association for Information Systems (AIS), 2010.

[66] M. L. Marmolejo and V. S. Ramírez, "Objetos de aprendizaje y ensenanza bibliotecologica. (Spanish)=", Ibersid, pp. 225-237, 2007.

[67] J. S. Lymn, F. Bath-Hextall, and H. J. Wharrad, "Pharmacology education for nurse prescribing students - a lesson in reusable learning objects", $B M C$ Nurs, vol. 7, p. 2, 2008.

[68] T. Taveira-Gomes, R. Prado-Costa, M. Severo, and M. A. Ferreira, "Characterization of medical students recall of factual knowledge using learning objects and repeated testing in a novel e-learning system", BMC Med Educ, vol. 15, p. 4, 2015.
[69] M. Beadle, Y. Needham, and M. Dearing, "Collaboration with service users to develop reusable learning objects: the ROOT to success", Nurse Educ Pract, vol. 12, pp. 352-5, Nov 2012.

[70] K. Rubinstein and M. E. Pittman, "Beyond PowerPoint (TM) : Using Learning Objects to Enhance Social Work Courses", Journal of Technology in Human Services, vol. 33, pp. 172-190, 2015.

[71] L. M. Tamashiro and H. H. Peres, "Development and assessment of learning objects about intramus. cular medication administration", Rev Lat Am Enfermagem, vol. 22 , pp. 716-723, Oct 2014

[72] R. Farrell, "Comics to the Rescue: Finding Innovative Applications for Library Digital Learning Objects", Journal of Library Innovation, vol. 5, pp. $67-77,2014$

[73] R. McGreal, Online education using learning objects. London - New York: Routledge Falmer, 2004.

[74] G. Ramírez, "Metodología para el Desarrollo y Producción de Objetos de Aprendizaje", in IX Congreso Nacional de Investigación Educativa. Consejo Mexicano de Investigación Educativa, Mérida, Yucatán, México, 2009.

[75] M. Borrero, E. Cruz, S. Mayorga, and K. Ramírez, Una metodología para el diseño de objetos de aprendizaje. La experiencia de la Dirección de Nuevas Tecnologías y Educación Virtual, DINTEV, [Online].. Available: http://objetos.univalle.edu.co/files/ articulo_AMED.pdf

[76] O. Suárez, L. Suárez, C. Sánchez, and M. Castilla, "Metodología para el diseño, desarrollo y evaluación de Objetos Virtuales de Aprendizaje", in Universidad Virtual 2008, La Habana, Cuba, 2008.

[77] M. Medina and M. López, "LOCoME: Metodología de Construcción de Objetos de Aprendizaje", presented at the III Simposio Pluridisciplinar sobre Diseño, Evaluación y Descripción de Contenidos Educativos Reutilizables (SPDECE), Oviedo, España, 2006.

[78] A. A. Silva-Sprock , P. G. , Julio-Cesar B, and Y. Hernández-Bieliukas, "Estado del Arte de las Metodologías para el Desarrollo de Objetos de Aprendizaje", in Conferencia Latino Americana de Objetos de Aprendizaje (LACLO), Valdivia, Chile, 2013.

[79] L. M. Campbell, "Evaluating 5/99 content for reusability as learning objects", VINE: The Journal of Information \& Knowledge Management Systems, vol. 35, pp. 85-96, 2005.

[80] X. Ochoa and E. Duval, "Estudio de las características de los repositorios de Objetos de Aprendizaje", in Tercera Conferencia Latinoamerica de Tecnología de Objetos de Aprendizaje (LACLO), Aguascalientes, México, 2008

[81] P. Castro-Martin, "Estadísticas de uso de repositorios institucionales: ¿un indicador fiable de comparación?", in $3^{\text {as }}$ Jornadas Os-Repositorios: La proyección de los repositorios institucionales, Madrid, España, 2008.

[82] M. López, A. Escalante, and S. Sánchez, "Reusabilidad de los Objetos de Aprendizaje almacenados en Repositorios de Libre Acceso", presented at the SPEDECE, Bilbao, España, 2007. 
[83] S. Ternier et al., "The Ariadne Infrastructure for Managing and Storing Metadata," Internet Computación, IEEE, vol. 3, n 4, pp. 18 - 25, 2009.

[84] Unión Europea, Proyecto Alter-nativa, 2016. [Online]. Available: https://www.udistrital.edu.co/novedades/particularNews.php?idNovedad=4396\& $\mathrm{Type}=\mathrm{N}$

[85] O. León, Referentes curriculares con incorporación de tecnologías para la formación del profesorado de matemáticas en y para la diversida, vol. 3. Bogotá, D.C.: Colombia: Universidad Distrital Francisco José de Caldas, 2014.
[86] N. Butcher, Guía Básica de Recursos Educativos Abiertos. París, Francia: Unesco, 2015.

[87] J. Duarte Duarte., "Ambientes de aprendizaje: una aproximación conceptual," Estud. pedagógicos, no. 29, pp. 97-113, 2003.

[88] G. Bachelard, El nuevo espíritu científico. México DF: Editorial Nueva Imagen, 1981. 